\title{
SISTEMA DE CONTROL DE POSICIÓN DEL ROTOR ESFÉRICO DE UN MOTOR ULTRASÓNICO
}

\author{
Antonio Morán Cárdenas
}

\section{Resumen}

El presente proyecto trata sobre el diseño del sistema de control de posición tridimensional del rotor esférico de un motor ultrasónico equipado con actuadores piezo-eléctricos. Los motores serán usados como articulaciones (hombro, rodilla) en exoesqueletos para soportar la caminata de ancianos y discapacitados.

Resultados experimentales muestran que la barra del rotor alcanza la posición espacial deseada, así como también sigue trayectorias deseadas aún en la presencia de perturbaciones externas. Estos resultados demuestran la capacidad de los motores ultrasónicos para ser usados en posicionamiento tridimensional con gran precisión, robustez y autonomía.

Palabras Clave: Motor ultrasónico, Rotor esférico, Control de posición angular, Actuador piezoeléctrico, Control en tiempo real.

\section{Abstract}

This project deals with the design of the control system for the accurate 3-dimensional positioning of the spherical rotor of an ultrasonic motor equipped with piezoelectric actuators. The motor is intended to be used to support human articulations (shoulder, knee) in exoskeletons for handicapped.

Experimental results show that the rotor bar achieves the desired spatial positions and follows desired trajectories with fast response and good accuracy even in the presence of external disturbances.

Keywords: Ultrasonic motor, Rotor ball, angular Attitude control, piezoelectric Actuator, Control in real time. 


\section{INTRODUCCIÓN}

Con la creación de nuevos materiales, hay un creciente interés por desarrollar nuevos tipos de motores y actuadores para diversas aplicaciones. Un nuevo tipo de actuador que ha despertado gran interés es el motor ultrasónico, que utiliza elementos piezo-eléctricos que transforman energía eléctrica en movimiento.

A diferencia de los motores eléctricos convencionales, los motores ultrasónicos no generan campos magnéti$\cos \mathrm{y}$, por lo tanto, pueden ser usados en equipos e instrumentos que no deben ser afectados por campos magnéticos, como es el caso de equipos e instrumentos médicos. Así también, los motores ultrasónicos ofrecen la posibilidad de trabajar con rotores que giran en una, dos o tres dimensiones, tal como es el caso de rotores esféricos que pueden girar en torno a diferentes ejes de rotación. Los motores ultrasónicos no requieren lubricación.

Dadas sus particulares características, los motores ultrasónicos vienen siendo usados en cámaras fotográficas, video-cámaras, brazos robóticos, equipos médicos, entre otras aplicaciones. En la Figura 1 se muestra un exoesqueleto equipado con motores ultrasónicos en las articulaciones y que es usado para mantener erguido el cuerpo de ancianos y discapacitados, a la vez que les permite caminar. Este proyecto fue desarrollado por la Universidad de Agricultura y Tecnología de Tokio, Japón, donde el autor formó parte del equipo investigador y tuvo a su cargo el diseño del sistema de control de posición angular de los motores ultrasónicos.

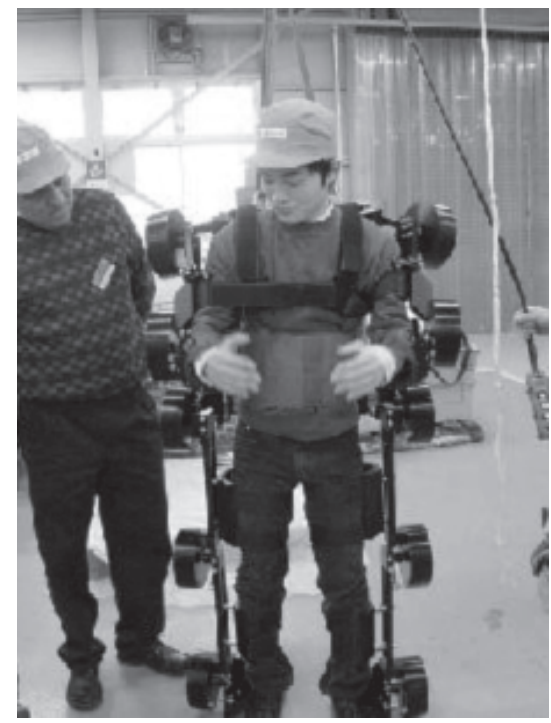

Figura 1. Exoesqueleto mecatrónico equipado con motores ultrasónicos. El autor aparece revisando el exoesqueleto desarrollado en la Universidad de Agricultura y Tecnologia de Tokio, Japón.

\section{OBJETIVOS}

El objetivo del presente proyecto es diseñar el sistema de control para el posicionamiento espacial y el seguimiento de trayectorias del rotor esférico de un motor ultrasónico. El sistema de control debe ser fácil de implementar en tiempo real y ofrecer un alto nivel de precisión en el posicionamiento del rotor, así como robustez ante la presencia de perturbaciones externas.

\section{MOTOR ULTRASÓNICO}

El motor ultrasónico es un novedoso tipo de actuador que usa vibracio- 
nes mecánicas en el rango ultrasónico (sobre los $20 \mathrm{KHz}$ ) como fuerza de tracción. Para producir las vibraciones, el motor usa cerámicos piezoeléctricos que se expanden y contraen (oscilan), de acuerdo a la magnitud del voltaje aplicado. Estos actuadores piezoeléctricos actúan como el estator del motor generando ondas viajeras que transmiten movimiento al rotor.

Según como los elementos piezoeléctricos son ubicados, se pueden configurar motores lineales o rotatorios. En la Figura 2, se muestra el estator de un motor ultrasónico con elementos piezoeléctricos distribuidos en un arreglo tipo disco. Al estator se le suministran dos señales de voltaje sinusoidal de la misma amplitud y frecuencia (cerca de $50 \mathrm{KHz}$ ), pero desfasadas en el tiempo. Variando la fase, el sentido de la rotación de las ondas viajeras generadas puede modificarse, para obtener diferentes patrones de movimiento del rotor.

En la Figura 3, se muestra la vista superior de un motor ultrasónico con rotor esférico usado en el presente proyecto. El motor tiene tres estatores tipo disco, separados 120 grados en un arreglo horizontal para soportar y transmitir movimiento al rotor esférico, al que se ha fijado una barra. Controlando la diferencia de fase de los voltajes aplicados a cada estator, el rotor esférico puede girar en cualquier dirección arbitraria (excepto el eje Z), y la barra puede ser posicionada apuntando hacia cualquier posición deseada o puede seguir una trayectoria deseada.

Los motores ultrasónicos presentan las siguientes ventajas: (a) baja influencia de campos magnéticos, (b) características de baja velocidad - alto torque, (c) torque de sujeción, (d) rápida respuesta, (e) estructura compacta, (f) operación sin ruido. En la actualidad, motores ultrasónicos unidimensionales son usados en cámaras fotográficas y video cámaras (para el control del zoom), en equipos de resonancia magnética, en vehículos de suspensión magnética, entre otras aplicaciones.

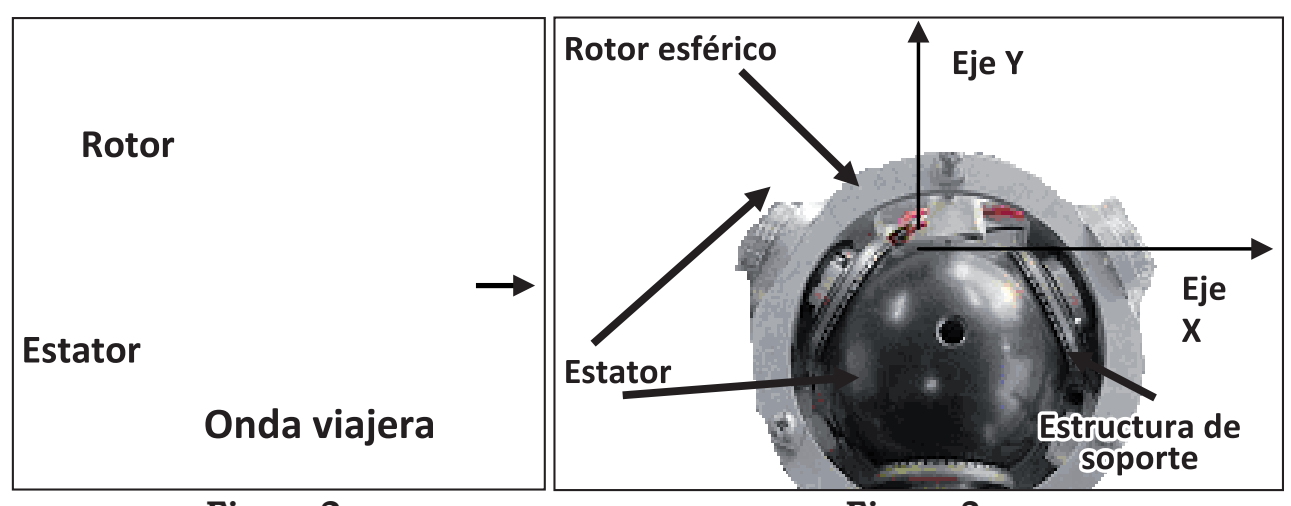

Figura 2.

Estator con ondas viajeras
Figura 3.

Motor ultrasónico o esférico 


\section{SISTEMA DE CONTROL DE POSICIÓN}

La estructura del motor ultrasónico y su sistema de control se muestra en la Figura 4. Se observan los tres estatores y el rotor esférico que tiene fija una barra. Para determinar la posición de la barra se usan dos codificadores ópticos; señales de salida se introducen a una computadora a través de un circuito contador para determinar los ángulos del rotor $\theta_{1}$ y $\theta_{2}$. Se ha propuesto una nueva estrategia de control que integra un algoritmo basado en relaciones geométricas en una estructura de control PID, para calcular el desfase del voltaje aplicado a cada estator y que están limitados al rango entre -90 y 90 grados.

Los desfases calculados por la computadora se envían a un driver de potencia a través de una tarjeta interfaz digital. El driver genera las señales de voltaje con el desfase requerido y los aplica a cada estator del motor cerrando el bucle de control realimentado. Una tarjeta reloj externa mantiene constante el tiempo de muestreo para el control en tiempo real.

La estructura general del sistema de control se muestra en la Figura 5. Las posiciones angulares, medidas $\theta_{1}$ y $\theta_{2}$, se comparan con las posiciones angulares deseadas $\mathrm{d}_{1} \mathrm{y} \mathrm{d}_{2}$ para determinar la dirección de rotación deseada del rotor. Para obtener la dirección de rotación, cada estator debe generar ondas viajeras con dirección e intensidad apropiadas. Esta dirección se determina a partir de la suma vectorial de los vectores correspondientes a la rotación de cada uno de los tres estatores. Posteriormente, la intensidad de cada vector se determina a través de un controlador PID, que aseguran un movimiento suave y preciso del rotor.

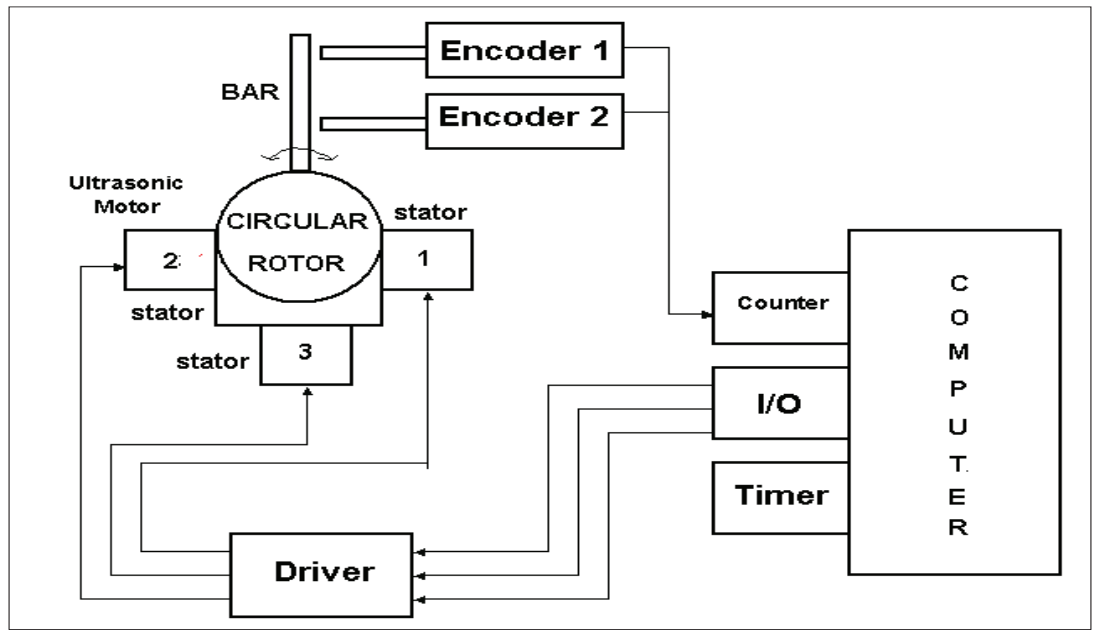

Figura 4. Diagrama de bloques del sisterna controlado 


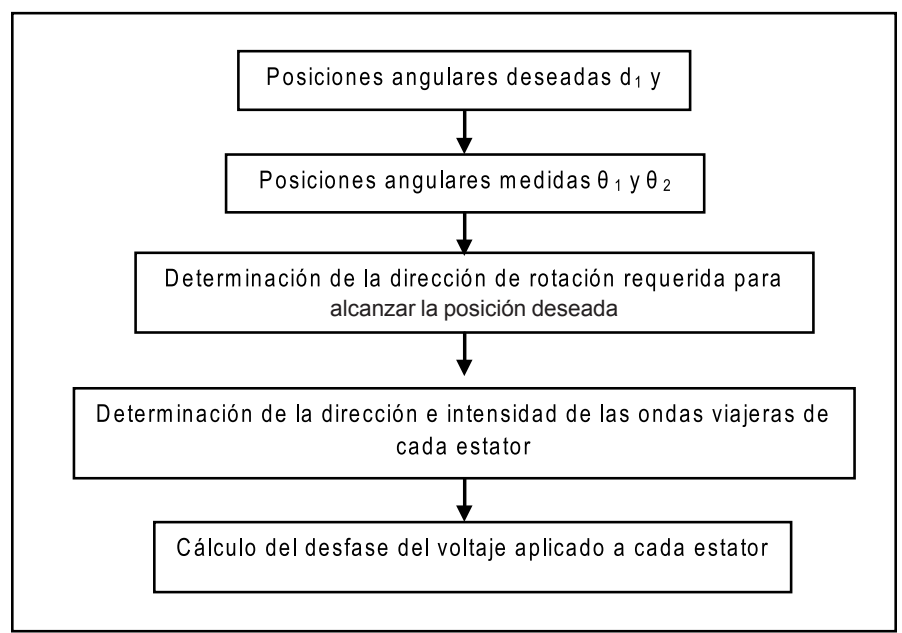

Figura 5. Estructura general de la estrategia

El proceso para calcular el desfase a aplicar a cada estator se muestra en la Figura 6. Se observa que, primero, se calculan los vectores de rotación normalizados de cada estator r1, r2 y r3; posteriormente, se usa un contro- lador PID para regular la intensidad de los vectores de rotación de cada estator, que son equivalentes al desfase del voltaje aplicado, que deben estar en el rango de -90 a 90 grados.

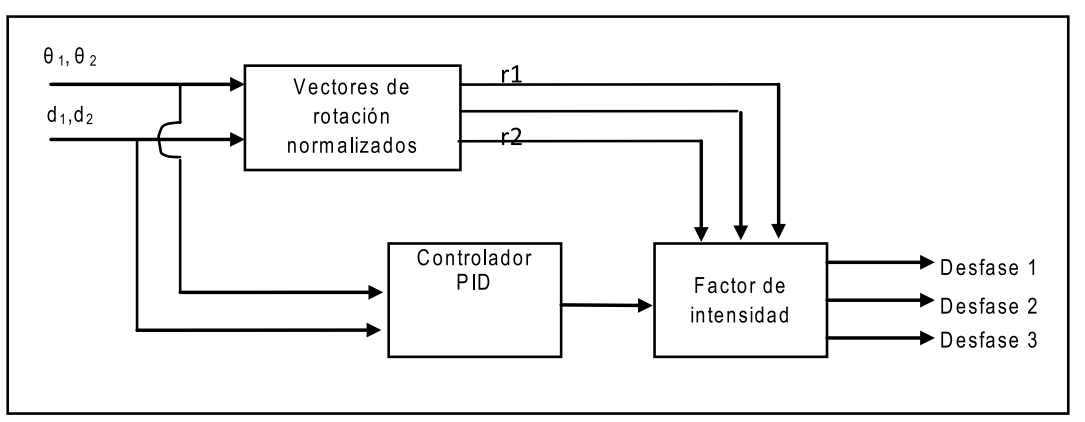

Figura 6. Cálculo del desfase de voltaje a aplicar a cada estator

\section{RESULTADOS EXPERIMENTALES}

Para verificar la eficacia de la estrategia de control propuesta, se han desarrollado varias pruebas experimentales de control de posición y de seguimiento de trayectorias deseadas de la barra fija al rotor esférico. Experimentalmente, se encontró que los valores adecuados de las ganancias del controlador PID son: $\mathrm{Kp}=90, \mathrm{Ki}=$ $600, \mathrm{Kd}=0$. 


\section{CONTROL DE POSICIÓN}

Con el objeto de analizar la respuesta transitoria del motor, se controló la rotación del rotor esférico, de manera que el extremo de la barra alcance diferentes posiciones fijas o variables a lo largo del eje $\mathrm{X}$.

En la Figura 7 (a), se muestra la respuesta de la posición de la barra del rotor para un valor deseado de 4 $\mathrm{mm}$, y, en la Figura 7 (b), se muestra el desfase del voltaje aplicado al esta-

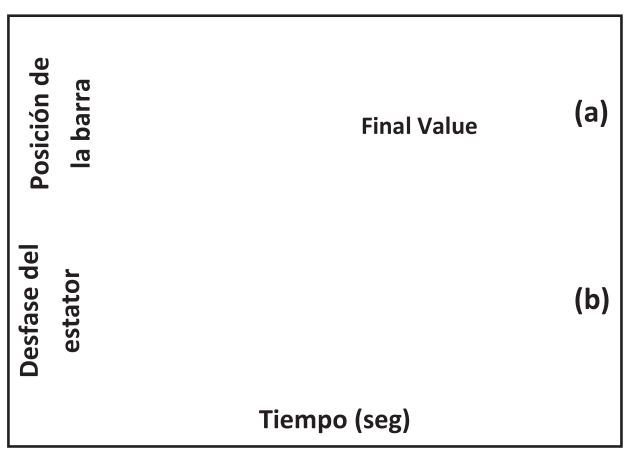

Figura 7. Respuesta para una entrada escalón de $4 \mathrm{~mm}$.
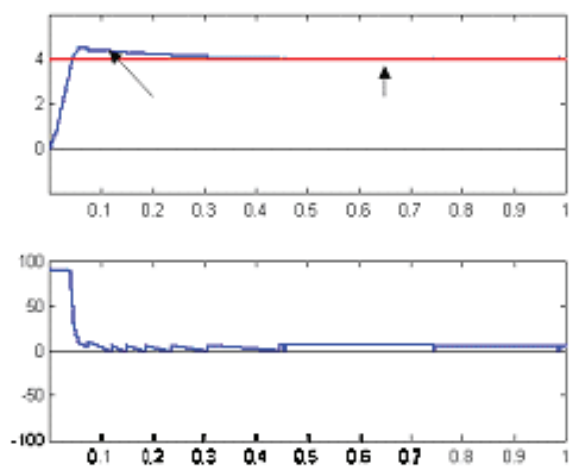

tor. Se puede notar que el rotor alcanza la posición deseada en menos de 0.3 segundos y $\sin$ error estacionario.

En la Figura 8 (a), se muestra la respuesta de la posición de la barra de rotor siguiendo una trayectoria sinusoidal deseada de $2 \mathrm{~Hz}$ de frecuencia y $5 \mathrm{~mm}$ de amplitud. Nuevamente se observa que el rotor sigue con gran precisión la trayectoria deseada. En la Figura 8(b,) se muestra el desfase del voltaje aplicado al estator, que debe estar en el rango de -90 a 90 grados.

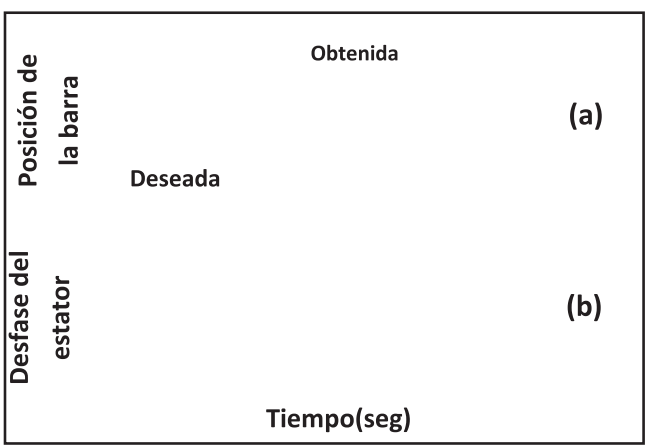

Figura 8. Respuesta para una entrada senoidal.
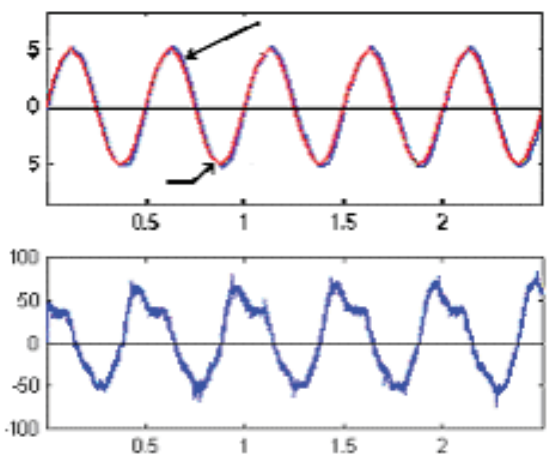


\section{CONTROL DE TRAYECTORIA}

La estrategia de control se aplicó de manera que el extremo de la barra fija al rotor esférico describa trayectoria de forma arbitraria. En la Figura 9, se muestra el desplazamiento de la barra siguiendo una trayectoria deseada cuadrada de $10 \mathrm{~mm}$. Se observa que el rotor sigue la trayectoria con un máximo error de +-, un pulso del codificador óptico que mide la posición angular del rotor. Resultados similares se

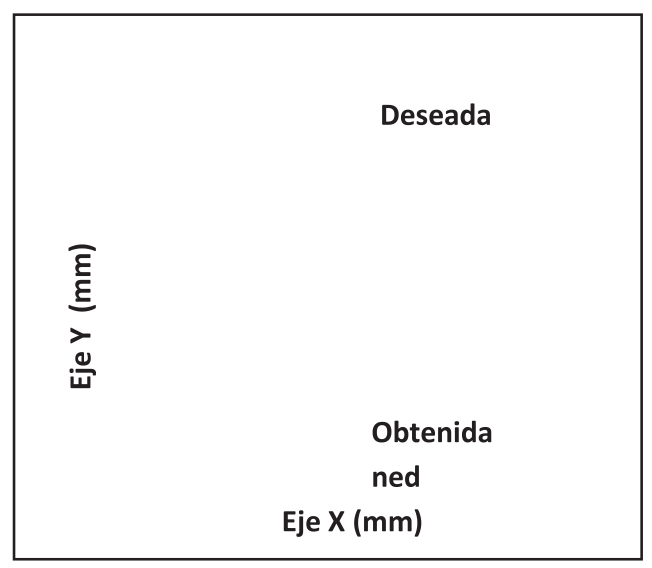

Figura 9. Seguimiento de trayectoria deseada.

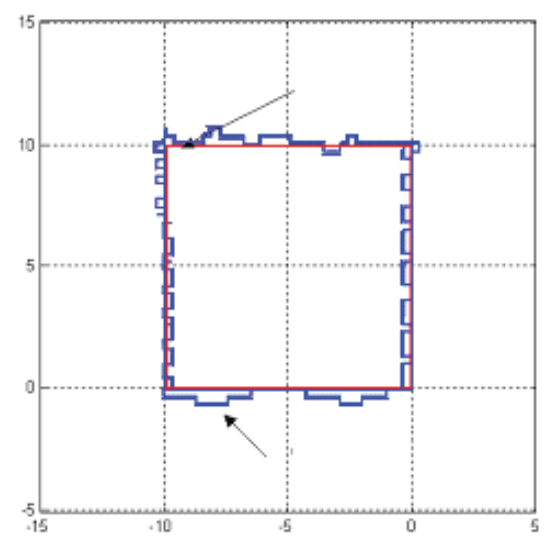

obtuvieron para trayectorias deseadas de forma poligonal.

Para verificar la robustez del sistema de control ante perturbaciones externas, el rotor se perturbó por una fuerza impulsiva externa, mientras que se movía describiendo una trayectoria cuadrada. Tal como se muestra en la Figura 10, el rotor es capaz de corregir el efecto negativo de la perturbación externa retornado a la trayectoria deseada.

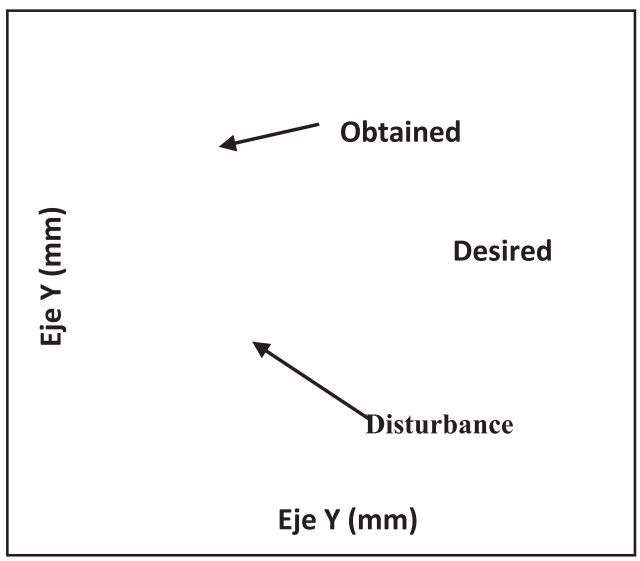

Figura 10. Efecto de perturbación externa.

Los resultados experimentales demuestran que la estrategia de control implementada permite al rotor alcanzar posiciones dadas y describir trayectorias deseadas con gran precisión y rapidez de respuesta, aun en la presencia de perturbaciones externas. Estos resultados demuestran la viabilidad de los motores ultrasónicos para el posicionamiento espacial y el seguimiento de trayectorias con precisión, robustez y autonomía. 


\section{CONCLUSIONES}

El motor ultrasónico es un novedoso actuador que usa vibraciones ultrasónicas producidas por elementos piezoeléctricos como fuerza motriz. El motor presenta características nolineales y de saturación de actuadores que lo convierten en un sistema complejo de controlar.

Se ha planteado una estrategia de control que no usa el modelo del motor, pero se basa en la integración de cálculos geométricos con un controlador PID para determinar los desfases del voltaje a aplicarse a cada estator.

Los resultados experimentales muestran que la barra fija al rotor esférico alcanza posiciones deseadas con precisión y rapidez, aún en la presencia de perturbaciones externas. Así también, se demostró que la barra puede describir trayectorias deseadas de forma espacial arbitraria. Estos resultados demuestran la capacidad y aplicabilidad de los motores ultrasónicos para desarrollar tareas de posicionamiento con precisión robustez y autonomía.

La investigación continúa para remplazar los codificadores ópticos por sensores de efecto Hall para determinar la posición angular de un rotor magnetizado. Esto permitirá reducir el tamaño del motor y de su sistema de medición. 


\section{REFERENCIAS BIBLIOGRÁFICAS}

Tomikawa, Y.; Ogasawara, T. \& Takano, T. (1989), Ultrasonic Motor Constructions / Characteristics / Applications.

Toshiku Sashida, Takashi Kenyo, (1993). An Introduction to Ultrasonic Motors, Oxford Press.

Ogata, Katsuhiko. (1998). Modern Control Engineering. Prentice Hall. 